\title{
A Canonical Trace Associated with Certain Spectral Triples ${ }^{\star}$
}

\author{
Sylvie PAYCHA
}

Laboratoire de Mathématiques, 63177 Aubière Cedex, France

E-mail: sylvie.paycha@math.univ-bpclermont.fr

URL: http://math.univ-bpclermont.fr/ paycha/

Received March 11, 2010, in final form September 13, 2010; Published online September 29, 2010 doi:10.3842/SIGMA.2010.077

\begin{abstract}
In the abstract pseudodifferential setup of Connes and Moscovici, we prove a general formula for the discrepancies of zeta-regularised traces associated with certain spectral triples, and we introduce a canonical trace on operators, whose order lies outside (minus) the dimension spectrum of the spectral triple.
\end{abstract}

Key words: spectral triples; zeta regularisation; noncommutative residue; discrepancies

2010 Mathematics Subject Classification: 58B34; 58J42; 47B47

\section{Introduction}

Connes and Moscovici's setup for abstract pseudodifferential calculus [4] (see also [6]) associated with a certain type of spectral triple $(\mathcal{A}, \mathcal{H}, D)$ provides a framework, in which the ordinary trace on trace-class operators can be extended to a linear form on all abstract pseudodifferential operators using zeta regualarisation type methods. Here, $\mathcal{A}$ is an involutive algebra represented in a complex Hilbert space $\mathcal{H}$ and $D$ a self-adjoint operator in $\mathcal{H}$. This linear extension, which depends on the operator $D$ used as a regulator in the zeta regularisation procedure, does not vanish on commutators as it can be seen from results of [4].

These constructions mimick the classical pseudodifferential calculus setup on a closed manifold, where similar linear extensions of the ordinary trace are built using the same zeta regularisation type procedure. On non-integer order operators, these linear extensions are independent of the regulator used in the zeta regularisation procedure. They define the canonical trace popularised by Kontsevich and Vishik [9], which vanishes on non-integer order commutators of classical pseudodifferential operators. The canonical trace is unique, in so far as any linear form on non-integer order classical pseudodifferential operators which vanishes on non-integer order commutators, is proportional to the canonical trace [12].

In the abstract pseudodifferential calculus framework, and under a mild reinforcement (see assumption $(\mathrm{H})$ in Section 4 and assumptions (Dk) and (T) in Section 5) of the usual assumptions on a regular spectral triple with discrete dimension spectrum $\mathrm{Sd}$ (used to define zeta regularised type linear extensions of the trace), we show that a similar canonical linear form on operators whose order lies outside the discrete set - Sd can be defined, and that this canonical linear form vanishes on commutators of operators, whose order lies outside the discrete set $-\mathrm{Sd}$.

The assumptions $(\mathrm{H}),(\mathrm{Dk})$ and $(\mathrm{T})$ we put on the spectral triple generalise the usual assumptions one puts on spectral triples to ensure the existence and a reasonable pole structure of meromorphic extensions of traces of holomorphic families of operators of the type $a|D|^{-z}$

\footnotetext{
*This paper is a contribution to the Special Issue "Noncommutative Spaces and Fields". The full collection is available at http://www.emis.de/journals/SIGMA/noncommutative.html
} 
with $a$ in $\mathcal{A}$. Our strengthened assumptions ensure the existence and a reasonable pole structure of meromorphic extensions of traces of more general holomorphic families $a(z)|D|^{-z}$ where $a(z)$ is a holomorphic family in $\mathcal{A} .{ }^{1}$ Broadly speaking, strengthening the assumptions on the spectral triple amounts to embedding the spectral triple $(\mathcal{A}, \mathcal{H}, D)$ into a holomorphic family $(\mathcal{A}(z), \mathcal{H}, D)$ of spectral triples such that $\mathcal{A}(0)=\mathcal{A}$. Under these strengthened assumptions, not only can one tackle traces of holomorphic families of the type $A|D|^{-z}$ where $A$ is an abstract pseudodifferential operator, but one can also deal with more general holomorphic families (see Definition 5 in Section 4)

$$
A(z) \simeq \sum_{j=0}^{\infty} b_{j}(z)|D|^{\alpha(z)-j}
$$

where $b_{j}(z)$ is a holomorphic family in the algebra $\mathcal{B}$ generated by elements of the type $\delta^{n}(a(z))$ or the type $\delta^{n}([D, a(z)])$ with $n$ varying in $\mathbb{Z}_{>0}$ and $a(z)$ any holomorphic family in $\mathcal{A}$.

We derive a general formula (see Theorem 1$)^{2}$

$$
\left.\operatorname{Res}_{0}^{j+1} \operatorname{Tr}(A(z))\right|^{\text {mer }}=\sum_{n=0}^{k-j} \frac{\tau_{j+n}^{|D|}\left(\left(\partial_{z}^{n}\left(A(z)|D|^{q z}\right)\right)_{\mid z=0}\right)}{q^{j+n+1} n !} \quad \forall j \in[[-1, k]],
$$

which expresses the complex residues at zero of the meromorphic extensions $\left.\operatorname{Tr}(A(z))\right|^{\text {mer }}$ of the trace $\operatorname{Tr}$ of a holomorphic family $A(z)$ in terms of residue type linear forms (see formula (5.1))

$$
\tau_{j}^{|D|}(A):=\left.\operatorname{Res}_{0}^{j+1} \operatorname{Tr}\left(A|D|^{-z}\right)\right|^{\text {mer }}
$$

introduced by Connes and Moscovici in [4]. Here $\left.\operatorname{Tr}\left(A|D|^{-z}\right)\right|^{\text {mer }}$ stands for the meromorphic extension of the holomorphic function $\operatorname{Tr}\left(A|D|^{-z}\right)$ defined on an adequately chosen half-plane.

Specialising to appropriate holomorphic families yields explicit formulae for the discrepancies of the linear extensions of the ordinary trace. With the help of a holomorphic family $(|D|+P)^{-z}$ defined in (6.1) where $P$ is a zero order perturbation, we can change the weight $|D|$ to $|D|+P$ in the linear forms $\tau_{j}^{|D|}$ in order to define the linear form $\tau_{j}^{|D|+P}$ (see formula (6.1)). The subsequent identity (see (6.2))

$$
\tau_{j}^{|D|+P}(A)-\tau_{j}^{|D|}(A)=\sum_{n=0}^{k-j} \frac{\tau_{j+n}^{|D|}\left(A\left(\partial_{z}^{n}\left((|D|+P)^{-z}|D|^{z}\right)_{\left.\right|_{z=0}}\right)\right)}{n !} \quad \forall j \in[[-1, k]],
$$

measures the sensitivity of $\tau_{j}^{|D|}$ to zero order perturbations of the weight $|D|$ and the following identity (see $(6.3))^{3}$

$$
\tau_{j}^{|D|}([A, B])=\sum_{n=0}^{k-j}(-1)^{n+1} \frac{\tau_{j+n}^{|D|}\left(A L^{n}(B)\right)_{\left.\right|_{z=0}}}{n !}, \quad \forall j \in[[-1, k]],
$$

which was derived in Proposition II.1 in [4] by other means, measures the obstruction to its vanishing on commutators.

\footnotetext{
${ }^{1} \mathrm{~A}$ family $\{f(z)\}_{z \in \Omega}$ in a topological vector space $E$, parametrised by a complex domain $\Omega$, is holomorphic at $z_{0} \in \Omega$ if the corresponding function $f: \Omega \rightarrow E$ is uniformly complex-differentiable on compact subsets in a neighborhood of $z_{0}$. When $f$ takes its values in a Banach space $E$, the existence of a complex derivative implies (via a Cauchy formula) that a holomorphic function is actually infinitely differentiable and admits a Taylor expansion which converges uniformly on a compact disk centered at $z_{0}$ (see e.g. [6, Theorem 8.1.7] and [5, Section XV.5.1]). Holomorphy of a Banach space valued function is therefore equivalent to its analyticity.

${ }^{2}$ We have set $[[a, b]]=[a, b] \cap \mathbb{Z}$ for two integers $a<b$, Res ${ }_{0}^{j}$ stands for the $j$-th order residue at zero and Res ${ }_{0}^{0}$ stands for the finite part at $z=0$.

${ }^{3}$ We have set $L(A):=[\log |D|, A]$.
} 
On the grounds of these identities, under assumptions $(\mathrm{H}),(\mathrm{Dk})$ and $(\mathrm{T})$ we show that the linear form $\tau_{-1}^{|D|}$ is invariant under zero order perturbations of $|D|$ (see Proposition 6) and that it vanishes on commutators for operators whose order lies outside minus the dimension spectrum (see Proposition 7). These remarkable features allow us to consider this linear form as a substitute in the abstract pseudodifferential operator set up for Kontsevish and Vishik's canonical trace.

To conclude, at the cost of embedding a spectral triple in a holomorphic family of spectral triples, we have built a linear form on operators whose order lies outside a given discrete set, which vanishes on commutators, whose orders lie outside that discrete set and which is insensitive to zero order perturbations of the weight $|D|$ used to build this linear form.

\section{Spectral triples and abstract differential operators}

We briefly recall the setup of abstract differential calculus as explicited in [6]. It encompasses essential features of ordinary pseudodifferential calculus on manifolds, as illustrated on a typical example throughout this paragraph.

Starting from a Hilbert space $\mathcal{H}$ with scalar product $\langle\cdot, \cdot\rangle$ and associated norm $\|\cdot\|$ together with a self-adjoint operator $D$ on $\mathcal{H}$, we build a non-negative self-adjoint operator $\Delta=D^{2}$. For simplicity, we assume the operator $\Delta$ is invertible and hence positive; if this is not the case we replace it with $1+\Delta$.

Example 1. Let $M$ be a closed and smooth spin Riemannian manifold, let $E$ be a Clifford Hermitian bundle over $M$ and $\nabla^{E}$ a Clifford connection on $E$. Let $C^{\infty}(M, E)$ denote the space of smooth sections of $E$ and its $L^{2}$-closure $\mathcal{H}=L^{2}(M, E)$ for the Hermitian product induced by the metric on $M$ combined with the Hermitian structure on the fibres of $E$. To this data corresponds a generalised Dirac operator (see e.g. [1])

$$
D=\sum_{i=1}^{n} c\left(d x_{i}\right) \nabla_{\frac{d}{d x_{i}}}^{E} .
$$

The self-adjoint operator $\Delta$ and its powers $\Delta^{k}$ are defined on dense domains $\operatorname{Dom}\left(\Delta^{k}\right)=$ $\operatorname{Dom}\left(D^{2 k}\right)$ in $\mathcal{H}$ and we consider the set

$$
\mathcal{H}^{\infty}:=\cap_{k=1}^{\infty} \operatorname{Dom}\left(\Delta^{k}\right)=\cap_{k=1}^{\infty} \operatorname{Dom}\left(D^{k}\right) .
$$

Example 2. With the notation of Example 1, the operators $\Delta^{k}=D^{2 k}$ acting on the space of smooth sections of $E$ are essentially self-adjoint and have unique self-adjoint extensions (denoted by the same symbol $\left.\Delta^{k}\right)$ to the $H^{k}$-Sobolev spaces $\operatorname{Dom}\left(\Delta^{k}\right)=H^{2 k}(M, E)$, obtained as closures of the space $C^{\infty}(M, E)$ of smooth sections of the bundle $E$ for the Sobolev norm

$$
\|u\|_{k}:=\left(\|u\|^{2}+\left\|\Delta^{\frac{k}{2}} u\right\|^{2}\right)^{\frac{1}{2}}
$$

(where $\|\cdot\|$ stands for the norm on $\mathcal{H}$ ), and whose intersection $\mathcal{H}^{\infty}$ coincides with $C^{\infty}(M, E)$.

We now introduce spectral triples [2], which are the building blocks for abstract pseudodifferential operators $[4,6]$.

Definition 1. A triple $(\mathcal{A}, \mathcal{H}, D)$, with $\mathcal{A}$ an involutive algebra represented in a complex Hilbert space $\mathcal{H}$ and $D$ a self-adjoint operator in $\mathcal{H}$, is a spectral triple, if it satisfies the following properties:

1) the operator $a(1+\Delta)^{-1}$ is compact for every element $a$ in $\mathcal{A}$,

2) the domain $\operatorname{Dom}(D)$ is stable under left multiplication by $\mathcal{A}$,

3) the adjoint operator $\delta_{D}: a \mapsto[D, a]$ on $\mathcal{A}$ extends to a bounded operator on $\mathcal{H}$. 
For any complex number $s$ we set $\Delta^{s}:=\int_{0}^{\infty} \lambda^{s} d P_{\lambda}$, where $P_{\lambda}:=1_{(-\infty, \lambda]}(\Delta)$ stands for the spectral projection of the self-adjoint operator $\Delta$ corresponding to the interval $(-\infty, \lambda]$. The space $\mathcal{H}^{s}$ is obtained as the completion of $\mathcal{H}^{\infty}$ for the norm ${ }^{4}$

$$
\|u\|_{s}:=\left\|\Delta^{\frac{s}{2 d}} u\right\| .
$$

If $\operatorname{Re}(s) \geq 0$, then $\mathcal{H}^{s}$ lies in $\mathcal{H}$ and coincides with the domain $\operatorname{Dom}\left(\Delta^{\frac{s}{2}}\right)$. If $\operatorname{Re}(s)<0$, then $\Delta^{\frac{s}{2}}$ is bounded on $\mathcal{H}$, whereas we have $\mathcal{H} \subsetneq \mathcal{H}^{s}$.

In the sequel, we shall often use the operator $|D|:=\sqrt{\Delta}$ and the unbounded derivation $\operatorname{ad}_{|D|}: X \mapsto[|D|, X]$ of the algebra $\mathcal{B}(\mathcal{H})$ of bounded linear operators on $\mathcal{H}$. Its domain is given by the set of bounded operators that map the domain of $|D|$ onto itself, and for which the commutator extends to a bounded operator on $\mathcal{H}$.

Definition 2. The spectral triple is said to be regular if and only if $\mathcal{A}$ and $[D, \mathcal{A}]$ belong to the domain of smoothness ${ }^{5}$

$$
\mathrm{Op}^{0}:=\cap_{n=1}^{\infty} \operatorname{Dom}\left(\delta^{n}\right),
$$

of the derivation $\delta$ on the algebra $\mathcal{B}(\mathcal{H})$.

Example 3. With the notation of Example 1, $\left(C^{\infty}(M, E), L^{2}(M, E), D\right)$ is a regular spectral triple.

For any complex number $s$, let $\mathrm{Op}^{s}$ be the set of operators defined by:

$$
P \in \mathrm{Op}^{s} \Longleftrightarrow|D|^{-s} P \in \mathrm{Op}^{0} .
$$

Let us also introduce the algebra ${ }^{6}$

$$
\mathcal{B}=\left\langle\delta^{n_{i}}\left(a_{i}\right), \delta^{n_{j}}\left(\left[D, a_{j}\right]\right), n_{i}, n_{j} \in \mathbb{Z}_{\geq 0}, a_{i}, a_{j} \in \mathcal{A}\right\rangle .
$$

For a regular spectral triple $(\mathcal{A}, \mathcal{H}, D)$, we have $\mathcal{B} \subset \mathrm{Op}^{0}$ and the operator $b|D|^{s}$ lies in $\mathrm{Op}^{s}$ for any $b$ in $\mathcal{B}$.

We henceforth assume that the spectral triple is regular and that $\mathcal{H}^{\infty}$ is stable under left multiplication by $\mathcal{A}$.

Following Higson [6] (see Definition 4.28 for the integer order case), for a complex number $a$ we consider the set $\mathcal{E}^{a}(\mathcal{A}, D)$ of operators $A: \mathcal{H}^{\infty} \rightarrow \mathcal{H}^{\infty}$, called basic pseudodifferential operators of order $a$, that have the following expansion:

$$
A \simeq \sum_{j=0}^{\infty} b_{j}|D|^{a-j}, \quad b_{j} \in \mathcal{B},
$$

meaning by this, that for any non-negative integer $N$

$$
R_{N}(A):=A-\sum_{j=0}^{N-1} b_{j}|D|^{a-j} \in \mathrm{Op}^{a-N} .
$$

Any finite linear combination of basic pseudodifferential operators of order $a$ is called an abstract pseudodifferential operator of order a. By Proposition 4.31 in [6] and Appendix B in [4], abstract pseudodifferential operators of integer order form an algebra filtered by the order, which is stable under the adjoint operator $\delta=[|D|, \cdot]$.

\footnotetext{
${ }^{4}$ Recall that $\Delta$ is assumed to be invertible; if this is not the case we use $1+\Delta$ instead.

${ }^{5}$ These notations are borrowed from [4], see Appendix B.

${ }^{6}$ Here $\langle\cdots\rangle$ means "generated by".
} 
Example 4. With the notation of Example 1, the triple consisting of $\mathcal{A}=C^{\infty}(M), \mathcal{H}=$ $L^{2}(M, E)$ and $D$ a generalised Dirac operator acting on $C^{\infty}(M, E)$ form a regular spectral triple. Abstract pseudodifferential operators of integer order correspond to a subalgebra of the algebra $C \ell^{\mathbb{Z}}(M, E)$ of classical pseudodifferential operators of integer order [7, 18, 19, 20], acting on smooth sections of the vector bundle $E$ over $M$. Since $D$ is a priori not an abstract pseudodifferential operator, the subalgebra corresponding to all integer order abstract pseudodifferential operators is a priori smaller than $C \ell^{\mathbb{Z}}(M, E)$.

By Lemma 4.30 in [6] (see also (11) in Part II of [4]), for any abstract pseudodifferential operator $A$ and any complex number $\alpha$ we have:

$$
|D|^{\alpha} A \simeq A|D|^{\alpha}+\sum_{k=1}^{\infty} c_{\alpha, k} \delta^{k}(A)|D|^{\alpha-k}
$$

where we have set $c_{\alpha, k}=\frac{\alpha(\alpha-1) \cdots(\alpha-k+1)}{k !}$ for any positive integer $k$. Note that if $A$ has order $a$, then $\delta^{k}(A)|D|^{\alpha-k}$ has order $a+\alpha-k$, whose real value decreases as $k$ increases.

Example 5. This formula holds for any classical pseudodifferential operator $A$ acting on smooth sections of a vector bundle $E \rightarrow M$ over a closed manifold $M$ and $D$ a generalised Dirac operator as in Example 1. In this case, the limit $\left[|D|^{\alpha}, A\right]=\lim _{N \rightarrow \infty} \sum_{k=1}^{N} c_{\alpha, k} \delta^{k}(A)|D|^{\alpha-k}$ holds in the Fréchet topology of classical pseudodifferential operators with constant order (see [9]), here $a+\alpha$ where $a$ is the order of $A$.

Definition 3. A spectral triple $(\mathcal{A}, \mathcal{H}, D)$ is finitely summable if for any element $a$ in $\mathcal{A}$, the operator $a|D|^{-1}$ lies in a Schatten class $\mathcal{L}^{p}(\mathcal{H})$ for some $1 \leq p<\infty$. Let $n$ stand for the infimum of the values $p$ for which this holds, called the degree of summability of $D$.

Remark 1. In particular, for any element $a$ in $\mathcal{A}$, the operator $a|D|^{-z}$ is trace-class on the half-plane $\operatorname{Re}(z)>n$. Holomorphicity of the map $z \mapsto \operatorname{Tr}\left(a|D|^{-z}\right)$ on the half-plane $\operatorname{Re}(z)>n$ then follows. Indeed, on any half-plane $\operatorname{Re}(z)>n+\epsilon$ for some positive $\epsilon$, the operator $a|D|^{-z}$ can be written as the product of a fixed trace-class operator $A:=a|D|^{-n-\epsilon}$ and a holomorphic family of bounded operators $B(z):=|D|^{-z+n+\epsilon}$ on that half-plane. Since holomorhicity implies analyticity for Banach spaces valued functions (see footnote 1), there are bounded operators $B_{n}$, $n \in \mathbb{Z}_{\geq 0}$ such that $B(z)=\sum_{n=0}^{\infty} B_{n} z^{n}$ converges uniformly on any compact disk centered at a point of the half-plane $\operatorname{Re}(z)>n+\epsilon$. Since $A$ is trace-class, so are the operators $A B_{n}$ and it follows that $\operatorname{Tr}\left(a|D|^{-z}\right)=\sum_{n=0}^{\infty} \operatorname{Tr}\left(A B_{n}\right) z^{n}$ is holomorphic on every half-plane $\operatorname{Re}(z)>n+\epsilon$ for positive $\epsilon$ and hence on the half-plane $\operatorname{Re}(z)>n$.

More generally, given a finitely summable regular spectral triple $(\mathcal{A}, \mathcal{H}, D)$ with degree of summability $n$, for any $A$ in $\mathcal{E}^{a}(\mathcal{A}, D)$, the map $\left.z \mapsto \operatorname{Tr}\left(A|D|^{-z}\right)\right|^{\text {mer }}$ is holomorphic on some half-plane $\operatorname{Re}(z-a)>n$ in $\mathbb{C}$ depending on the order $a$ of $A$.

Definition 4. A regular and finitely summable spectral triple has discrete dimension spectrum, if there is a discrete subset $S \subset \mathbb{C}$ such that for any operator $A \in \mathcal{E}^{a}(\mathcal{A}, D)$ with order $a$, the map $z \mapsto \operatorname{Tr}\left(A|D|^{-z}\right)$ extends to a meromorphic map $\left.z \mapsto \operatorname{Tr}\left(A|D|^{-z}\right)\right|^{\text {mer }}$ on $\mathbb{C}$ with poles in the set $S-a$. Let $\mathrm{Sd}$ denote the smallest such set $S$ called the dimension spectrum.

The dimension spectrum is simple if all the poles are simple. It has finite multiplicity $k \in \mathbb{N}$ if the poles are at most of order $k$. 
Example 6. With the notation of Example 1, let $A$ be a classical pseudodifferential operator of real order $a$, acting on $C^{\infty}(M, E)$. The operator $z \mapsto \operatorname{Tr}\left(A|D|^{-z}\right)$ is holomorphic on the half-plane $\operatorname{Re}(z)>a+n$, where $n$ is the dimension of the underlying manifold $M$, and has a meromorphic extension to the whole complex plane with simple poles in $]-\infty, a+n] \cap \mathbb{Z}$ (see [17]). This meromorphic extension can be written in terms of the canonical trace popularised by Kontsevich and Vishik [9] (see also [10])

$$
z \mapsto \operatorname{TR}\left(A|D|^{-z}\right)
$$

The regular spectral triple $(\mathcal{A}, \mathcal{H}, D)$ arising from Example 1 therefore has a discrete simple dimension spectrum given by $\mathrm{Sd}=]-\infty, n] \cap \mathbb{Z}$, which is stable under translations by negative integers. Note that the meromorphic extension is holomorphic at zero if the order $a$ is noninteger.

\section{$3 \quad$ Logarithms}

Given a regular spectral triple ${ }^{7}(\mathcal{A}, \mathcal{H}, D)$, the logarithm being a continuous function on the spectrum of the operator $|D|=\sqrt{\Delta}$, one can define the unbounded self-adjoint operator $\log |D|$ by Borel functional calculus. It can also be viewed as the derivative at zero of the complex power $|D|^{z}$. For any positive $\epsilon$, the map $z \mapsto|D|^{z-\epsilon} \in \mathrm{Op}^{z-\epsilon}$ defines a holomorphic function on the half plane $\operatorname{Re}(z)<\epsilon$ with values in $\mathcal{B}(\mathcal{H})$ and we have $\log |D|=|D|^{\epsilon}\left(\partial_{z}\left(|D|^{z-\epsilon}\right)\right)_{\left.\right|_{z=0}}$. For any positive $\epsilon$, the operator $\log |D||D|^{-\epsilon}=|D|^{-\epsilon} \log |D|$ lies in $\mathrm{Op}^{0}$, so that $\log |D|$ lies in $\mathrm{Op}^{\epsilon}$ for any positive $\epsilon$.

For any complex number $a$ and any positive integer $L$, we now introduce the set $\mathcal{E}^{a, L}(\mathcal{A}, D)$ of operators $A: \mathcal{H}^{\infty} \rightarrow \mathcal{H}^{\infty}$, which have the following expansion:

$$
A \simeq \sum_{l=0}^{L} \sum_{j=0}^{\infty} b_{j, l}|D|^{\alpha-j} \log ^{l}|D|, \quad b_{j, l} \in \mathcal{B}
$$

meaning by this that for any non-negative integer $N$

$$
R_{N}(A):=A-\sum_{l=0}^{L} \sum_{j=0}^{N-1} b_{j, l}|D|^{\alpha-j} \log ^{l}|D| \in \mathrm{Op}^{\alpha-N+\epsilon}, \quad \forall \epsilon>0 .
$$

By convention we set $\mathcal{E}^{\alpha, 0}(\mathcal{A}, D)=\mathcal{E}^{\alpha}(\mathcal{A}, D)$.

Remark 2. In the setup of pseudodifferential operators acting on smooth sections of a vector bundle over a closed manifold $M$, for an operator $D$ as in Example 1, the set $\mathcal{E}^{a, L}(\mathcal{A}, D)$ is reminiscent of the set $C \ell^{a, L}(M, E)$ of log-polyhomogeneous operators of order $a$ and logarithmic type $L$ investigated in [10]. Setting $L=0$ yields back classical pseudodifferential operators.

We call a linear operator $A: \mathcal{H}^{\infty} \rightarrow \mathcal{H}^{\infty}$ in $\mathcal{E}^{a, L}(\mathcal{A}, D)$, a basic pseudodifferential operator of order a and logarithmic type $L$. An abstract pseudodifferential operator of order $a$ and logarithmic type $L$ is a finite linear combination of basic abstract pseudodifferential operators of order $a$ and logarithmic type $L$.

Setting $a=z$ in (2.1) and differentiating with respect to $z$ at zero yields

$$
\log |D| A \simeq A \log |D|+\sum_{k=1}^{\infty} c_{0, k}^{\prime} \delta^{k}(A)|D|^{-k},
$$

\footnotetext{
${ }^{7}$ Recall that $\Delta$ is assumed to be invertible; otherwise we replace $\Delta$ with $\Delta+1$.
} 
for any abstract pseudodifferential operator $A$, with $c_{0, k}^{\prime}:=\left.\partial_{z} c_{z, k}\right|_{z=0}=\sum_{j=1}^{k-1} \frac{(-1)^{j}}{j}$ for positive integers $k$. In particular, this implies that the logarithmic type does not increase by the adjoint action with $\log |D|$, so that for any complex number $a$ and any integer $k$, we have

$$
A \in \mathcal{E}^{a, k}(\mathcal{A}, D) \Longrightarrow[\log |D|, A] \in \mathcal{E}^{a-1, k}(\mathcal{A}, D) .
$$

Remark 3. This is reminiscent of the fact that in the classical setup, the bracket of the logarithms of an elliptic operator (with appropriate conditions on the spectrum for the logarithm to be defined) with a classical pseudodifferential operator, is classical in spite of the fact that the logarithm is not classical.

The following proposition compares the logarithms of two operators of the same order.

Proposition 1. For any $P \simeq \sum_{j=0}^{\infty} b_{j}|D|^{-j} \in \mathcal{E}^{0}(\mathcal{A}, D)$, the logarithm $\log (|D|+P)$ is well-defined and lies in $\mathcal{E}^{0,1}(\mathcal{A}, D)$. More precisely, the difference $\log (|D|+P)-\log |D|$ of the two logarithms lies in $\mathcal{E}^{0}(\mathcal{A}, D)$.

Proof. By (2.1) applied to $\alpha=-1$, the operator $|D|^{-1} P$ is an abstract pseudodifferential operator of order -1 and hence a bounded operator on $\mathcal{H}$. The operator $\log \left(1+|D|^{-1} P\right)$ defined by Borel functional calculus reads:

$$
\log \left(1+|D|^{-1} P\right) \simeq \sum_{i=0}^{\infty} \frac{(-1)^{i-1}}{i !}\left(|D|^{-1} P\right)^{i},
$$

and hence lies in $\mathcal{E}^{0}(\mathcal{A}, D)$ by $(2.1)$.

The Campbell-Hausdorff formula (see [13] in the classical case) then yields:

$$
\left.\log (|D|+P)-\log |D| \simeq \log \left(1+|D|^{-1} P\right)+\sum_{k=2}^{\infty} C^{(k)}\left(\left(\log |D|, 1+|D|^{-1} P\right)\right)\right),
$$

where $C^{(k)}(\cdot, \cdot)$ are Lie monomials given by:

$$
C^{(k)}(A, B):=\sum_{j=1}^{\infty} \frac{(-1)^{j+1}}{(j+1)} \sum \frac{\left(\operatorname{ad}_{A}\right)^{\alpha_{1}}\left(\operatorname{ad}_{B}\right)^{\beta_{1}} \cdots\left(\operatorname{ad}_{A}\right)^{\alpha_{j}}\left(\operatorname{ad}_{B}\right)^{\beta_{j}} B}{\left(1+\sum_{l=1}^{j} \beta_{l}\right) \alpha_{1} ! \cdots \alpha_{j} ! \beta_{1} ! \cdots \beta_{j} !},
$$

with the inner sum running over $j$ - tuples of pairs $\left(\alpha_{i}, \beta_{i}\right)$ such that $\alpha_{i}+\beta_{i}>0$ and $\sum_{i=1}^{j} \alpha_{i}+\beta_{i}=k$, and with the following notational convention: $\left(\operatorname{ad}_{A}\right)^{\alpha_{j}}\left(\operatorname{ad}_{B}\right)^{\beta_{j}-1} B=\left(\operatorname{ad}_{A}\right)^{\alpha_{j}-1} A$ if $\beta_{j}=0$, in which case this vanishes if $\alpha_{j}>1$. Here $\operatorname{ad}_{X}:=[X, \cdot]$ denotes the adjoint action by an operator $X$. Property (3.1) applied to $A=\log \left(1+|D|^{-1} P\right)$, yields that

$$
C^{(1)}\left(\log |D|, \log \left(1+|D|^{-1} P\right)\right)=\frac{1}{2}\left[\log |D|, \log \left(1+|D|^{-1} P\right)\right]
$$

lies in $\mathcal{E}^{0}(\mathcal{A}, D)$. By induction on $k$, one shows that $C^{(k)}\left(\log |D|, \log \left(1+|D|^{-1} P\right)\right)$ lies in $\mathcal{E}^{0}(\mathcal{A}, D)$ for any positive integer $k$, so that the difference $\log (|D|+P)-\log |D|$ also lies in $\mathcal{E}^{0}(\mathcal{A}, D)$.

Note that by (2.1) the adjoint operation $\operatorname{ad}_{\log \left(1+|D|^{-1} B\right)}$ decreases the order by 1 unit and that by (3.1) the same property holds for the adjoint operation $\operatorname{ad}_{\log |D|}$.

Remark 4. Proposition 1 is reminiscent of the fact that in the classical setup, the difference of the logarithms of two elliptic operators (with appropriate conditions on the spectrum for their logarithms to be defined) is classical in spite of the fact that each logarithm is not classical. 


\section{Holomorphic families of operators}

Let $(\mathcal{A}, \mathcal{H}, D)$ be a regular spectral triple with discrete finite dimension spectrum Sd and degree of summability $n$. Under the regularity assumption, one can equip $\mathcal{A}$ with a locally convex topology by means of semi-norms $a \mapsto\left\|\delta^{k}(a)\right\|$ and $a \mapsto\left\|\delta^{k}([D, a])\right\|$. The completion of $\mathcal{A}$ for this topology is a Fréchet space [21] (and even a Fréchet $C^{*}$-algebra), which we denote by the same symbol $\mathcal{A}$, so that we shall henceforth assume that $\mathcal{A}$ is a Fréchet space. Equivalence between holomorphicity and analyticity for Banach space valued functions (see footnote 1) actually extends to the case of Fréchet valued functions; the proof in the Banach case, which uses the Cauchy formula indeed extends to a Fréchet space in replacing the norm on the Banach space by each of the semi-norms that define the topology on the Fréchet space.

We call a $\mathcal{B}$-valued function $b(z)$ holomorphic, if it is a linear combination of products of operators $\delta^{n_{i}}\left(a_{i}(z)\right)$ and $\delta^{n_{j}}\left(\left[|D|, a_{j}(z)\right]\right)$ for some non-negative integers $n_{i}$ and $n_{j}$, where $a_{i}(z)$ and $a_{j}(z)$ are holomorphic families in $\mathcal{A}$.

- Assumption (H). For any holomorphic family $z \mapsto b(z)$ in $\mathcal{B}$ the map $z \mapsto \operatorname{Tr}\left(b(z)|D|^{-z}\right)$ is holomorphic on the half plane $\operatorname{Re}(z)>n$ and extends to a meromorphic map

$$
\left.z \mapsto \operatorname{Tr}\left(b(z)|D|^{-z}\right)\right|^{\text {mer }}
$$

with poles in the same set $\mathrm{Sd}$.

Example 7. With the notation Example 1, the spectral triple $\left\{C^{\infty}(M), L^{2}(M, E), D\right\}$ satisfies Assumption $(\mathrm{H})$. This follows from the fact that for any holomorphic family $z \mapsto f(z)$ in $C^{\infty}(M)$, seen as a family of multiplication operators, the map $z \mapsto \operatorname{Tr}\left(f(z)\left(1+D^{2}\right)^{-z / 2}\right)$ has a meromorphic extension $z \mapsto \operatorname{TR}\left(f(z)\left(1+D^{2}\right)^{-z / 2}\right)$ with simple poles in the set $\left.\left.\mathbb{Z} \cap\right]-\infty, n\right]$ and where, as before, TR stands for the canonical trace.

This is actually a particular instance (with $A(z)=f(z)\left(1+D^{2}\right)^{-z / 2}$ ) of the following more general result, which can be found in [9] (see also [14] for a review). For any holomorphic family of classical pseudodifferential operators $A(z)$ with non-constant affine order $\alpha(z)$ acting on $C^{\infty}(M, E)$, the map $z \mapsto \mathrm{TR}(A(z))$ is meromorphic with simple poles in the discrete set $\alpha^{-1}(\mathbb{Z} \cap[-n,+\infty[)$. It is therefore natural to introduce the following definition inspired from the notion of holomorphic family of operators used by Kontsevich and Vishik in [9].

Definition 5. We call a family

$$
A(z) \simeq \sum_{j=0}^{\infty} b_{j}(z)|D|^{\alpha(z)-j} \in \mathcal{E}^{\alpha(z)}(\mathcal{A}, D)
$$

of linear operators acting on $\mathcal{H}^{\infty}$ parametrised by $z \in \mathbb{C}$, holomorphic, if

1) $\alpha(z)$ is a complex valued holomorphic function,

2) $b_{j}(z)$ is a $\mathcal{B}$-valued holomorphic function for any non-negative integer $j$,

3) the family $A(z)|D|^{-\alpha(z)}$ is holomorphic in the Banach space $\mathcal{B}(\mathcal{H})$ and

$$
\partial^{(k)}\left(A(z)|D|^{-\alpha(z)}\right) \simeq \sum_{j=0}^{\infty} b_{j}^{(k)}(z)|D|^{-j} \in \mathcal{E}^{0}(\mathcal{A}, D) .
$$

Example 8. For any complex number $a$ and any operator $A$ in $\mathcal{E}^{a}(\mathcal{A}, D)$, the family $A(z)=$ $A|D|^{-z}$ is a holomorphic family of order $\alpha(z)=a-z$ acting on $\mathcal{H}^{\infty}$. 
The following lemma extends this to families $A(z)=|D|^{-z} A$.

Lemma 1. For any complex number $a$ and any operator $A \simeq \sum_{j=0}^{\infty} b_{j}|D|^{a-j} \in \mathcal{E}^{a}(\mathcal{A}, D)$, the product $|D|^{-z} A$ is a holomorphic family of order $a-z$ acting on $\mathcal{H}^{\infty}$, and the commutator $\left[|D|^{-z}, A\right]$ is a holomorphic family of order $a-z-1$ acting on $\mathcal{H}^{\infty}$.

Proof. Applying (2.1), with $\alpha$ replaced with $-z$, to the operators $b_{j}|D|^{a-j}$ for any non-negative integer $j$, yields

$$
|D|^{-z} A \simeq \sum_{k=0}^{\infty} c_{-z, k} \sum_{j=0}^{\infty} \delta^{k}\left(b_{j}\right)|D|^{a-j-k-z} \simeq \sum_{i=0}^{\infty}\left(\sum_{j=0}^{i} c_{-z, i-j} \delta^{i-j}\left(b_{j}\right)\right)|D|^{a-z-i} .
$$

We shall also need the following technical result.

Proposition 2. Given a holomorphic family $A(z) \simeq \sum_{j=0}^{\infty} b_{j}(z)|D|^{\alpha(z)-j}$ acting on $\mathcal{H}^{\infty}$, of non constant affine order $\alpha(z)=a-q z$, the higher derivatives $\partial_{z}^{(k)}\left(A(z)|D|^{-\alpha(z)}\right)$ lie in $\mathcal{E}^{0}(\mathcal{A}, D)$, whereas the higher derivatives $\partial_{z}^{(k)} A(z)$ lie in $\mathcal{E}^{\alpha(z), k}(\mathcal{A}, D)$.

Proof. By assumption, the family $A(z)|D|^{-\alpha(z)}$ is holomorphic in the Banach space $\mathcal{B}(\mathcal{H})$, with derivatives

$$
\partial_{z}^{k}\left(A(z)|D|^{-\alpha(z)}\right) \simeq \sum_{j=0}^{\infty} \partial_{z}^{k} b_{j}(z)|D|^{-j}
$$

in $\mathcal{E}^{0}(\mathcal{A}, D)$. Since $|D|^{\alpha(z)}$ is a holomorphic family with derivatives

$$
\partial_{z}^{l}\left(|D|^{\alpha(z)}\right)=(-q)^{l} \log ^{l}|D||D|^{\alpha(z)}
$$

that lie in $\mathcal{E}^{\alpha(z), l}(\mathcal{A}, D)$, the Leibniz rule yields the following identity of (unbounded) operators:

$$
A^{(k)}(z)=\sum_{l=0}^{k}\left(\begin{array}{l}
k \\
l
\end{array}\right)(-q)^{l}\left(\sum_{j=0}^{\infty} \partial_{z}^{k-l} b_{j}(z)|D|^{\alpha(z)-j}\right) \log ^{l}|D| \in \mathcal{E}^{\alpha(z), k}(\mathcal{A}, D) .
$$

Example 9. For any complex number $a$ and an operator $A \in \mathcal{E}^{a}(\mathcal{A}, D)$, the bracket $\left[A,|D|^{-z}\right]$ and the difference $A\left((|D|+P)^{-z}-|D|^{-z}\right)$ define holomorphic families acting on $\mathcal{H}^{\infty}$. By the results of the previous section, their derivatives at zero $-[A, \log |D|]$ and $A(\log |D|-\log (|D|+P))$, which are expected to be in $\mathcal{E}^{a, 1}(\mathcal{A}, D)$ actually lie in $\mathcal{E}^{a}(\mathcal{A}, D)$.

\section{Traces of holomorphic families}

Let $(\mathcal{A}, \mathcal{H}, D)$ be a regular spectral triple which satisfies assumption $(\mathrm{H})$ of the previous section. We make two further assumptions

- Assumption (Dk). The poles of the meromorphic map $\left.z \mapsto \operatorname{Tr}\left(b(z)|D|^{-z}\right)\right|^{\text {mer }}$ introduced in (4.1) have multiplicity $\leq k+1$.

- Assumption (T). The dimension spectrum Sd is invariant under translation by negative integers:

$$
\mathrm{Sd}-\mathbb{N} \subset \mathrm{Sd} .
$$


Remark 5. Under assumption (Dk) we have

$$
\operatorname{Res}_{0}^{j}\left(\left.\operatorname{Tr}\left(b(z)|D|^{-z}\right)\right|^{\text {mer }}\right)=0 \quad \forall j>k+1,
$$

where for a meromorphic function $f$ with a pole of order $N$ at zero we write in a neighborhood of zero:

$$
f=: \sum_{j=0}^{N} \frac{\operatorname{Res}_{0}^{j} f}{z^{j}}+O(z) .
$$

Example 10. Results of Kontsevich and Vishik [9] tell us that assumptions $(D k)$ and $(T)$ are satisfied in the classical geometric setup corresponding to Example 1. Indeed, assumption $(D k)$ is satisfied for $k=0$ since $\operatorname{TR}\left(b(z)|D|^{-z}\right)$ is a meromorphic map with simple poles. These lie in the set $]-\infty,-n] \cap \mathbb{Z}$, which is stable under translation by negative integers so that assumption $(\mathrm{T})$ is satisfied.

We henceforth assume that $(\mathrm{H}),(\mathrm{Dk})$ and $(\mathrm{T})$ are satisfied.

Proposition 3. Let $A(z) \simeq \sum_{j=0}^{\infty} b_{j}(z)|D|^{\alpha(z)-j}$ be a holomorphic family of operators acting on $\mathcal{H}^{\infty}$ with non-constant affine order $\alpha(z)$. The map $z \mapsto \operatorname{Tr}(A(z))$ is well-defined and holomorphic on the half plane $\operatorname{Re}(\alpha(z))<-n$, and further extends to a meromorphic function $\left.\operatorname{Tr}(A(z))\right|^{\text {mer }}$ on the complex plane with poles in $\alpha^{-1}(-\mathrm{Sd})$ with multiplicity $\leq k+1$.

Proof. Let us set $\alpha(z):=-q z+a$ for some positive real number $q$ (a similar proof holds for negative $q$ ). Under assumption $(\mathrm{H})$, for any non-negative integer $j$ the map

$$
z \mapsto \operatorname{Tr}\left(b_{j}(z)|D|^{\alpha(z)-j}\right)=\operatorname{Tr}\left(b_{j}(z)|D|^{-q\left(z-\frac{a-j}{q}\right)}\right)
$$

is holomorphic on the half-plane

$$
\operatorname{Re}(z)>\frac{\operatorname{Re}(a)+n-j}{q} \Longleftrightarrow \operatorname{Re}(\alpha(z))<-n+j .
$$

Under Assumption (Dk), it extends to a meromorphic map $\left.\operatorname{Tr}\left(b_{j}(z)|D|^{-q\left(z-\frac{a-j}{q}\right)}\right)\right|^{\text {mer }}$ with poles of multiplicity $\leq k+1$ in the set

$$
\frac{\mathrm{Sd}+a-j}{q} \subset \frac{\mathrm{Sd}+a}{q}=\alpha^{-1}(-\mathrm{Sd})
$$

as a consequence of Assumption ( $\mathrm{T})$.

On the other hand, given a real number $r$ and an integer $N>\operatorname{Re}(a)+n-r q$, under Assumption (H), the map $z \mapsto \operatorname{Tr}\left(K_{N}(z)\right)$ is holomorphic on the half-plane $\operatorname{Re}(z)>r$, where

$$
K_{N}(z):=A(z)-\sum_{j=0}^{N-1} b_{j}(z)|D|^{\alpha(z)-j} \simeq \sum_{j=N}^{\infty} b_{j}(z)|D|^{\alpha(z)-j}
$$

is the remainder operator. Combining these observations yields a meromorphic map on the half-plane $\operatorname{Re}(z)>r$ given by

$$
\left.\operatorname{Tr}(A(z))\right|^{\text {mer }}:=\left.\sum_{j=0}^{N-1} \operatorname{Tr}\left(b_{j}(z)|D|^{\alpha(z)-j}\right)\right|^{\text {mer }}+\operatorname{Tr}\left(K_{N}(z)\right),
$$

with poles in $\frac{\mathrm{Sd}+a}{q}=\alpha^{-1}(-\mathrm{Sd})$ with multiplicity $\leq k+1$. Since $r$ can be chosen arbitrarily, this provides a meromorphic extension to the whole complex plane with poles in $\alpha^{-1}(-\mathrm{Sd})$ with multiplicity $\leq k+1$. The result then follows. 
Using the notation of [4], we set the following definition.

Definition 6. For an abstract pseudodifferential operator $A$ and an integer $j$ in $[-1, \infty[$, let

$$
\tau_{j}^{|D|}(A):=\left.\operatorname{Res}_{0}^{j+1} \operatorname{Tr}\left(A|D|^{-z}\right)\right|^{\text {mer }} .
$$

If $j=-1$, this amounts to the finite part at $z=0$ :

$$
\tau_{-1}^{|D|}(A)=\left.\mathrm{fp}_{z=0} \operatorname{Tr}\left(A|D|^{-z}\right)\right|^{\text {mer }} .
$$

Remark 6. Under assumption (Dk), we have that $\tau_{j}^{|D|}(A)=0 \forall j>k$.

The following statement extends Proposition II.1 in [4].

Theorem 1. Let $A(z) \simeq \sum_{j=0}^{\infty} b_{j}(z)|D|^{\alpha(z)-j}, b_{j}(z) \in \mathcal{B}$, be a holomorphic family of non-constant affine order $\alpha(z)=a-q z$ for some positive $q$.

1. For any integer $j \in[[-1, k]]:=\mathbb{Z} \cap[-1, k]$, the $(j+1)$-th residue reads

$$
\left.\operatorname{Res}_{0}^{j+1} \operatorname{Tr}(A(z))\right|^{\text {mer }}=\sum_{n=0}^{k-j} \frac{\tau_{j+n}^{|D|}\left(\left(\partial_{z}^{n}\left(A(z)|D|^{q z}\right)\right)_{\mid z=0}\right)}{q^{j+n+1} n !} .
$$

2. In particular,

$$
\mathrm{fp}_{z=0}\left(\left.\operatorname{Tr}(A(z))\right|^{\mathrm{mer}}\right)=\sum_{n=0}^{k+1} \frac{\tau_{n-1}^{|D|}\left(\left(\partial_{z}^{n}\left(A(z)|D|^{q z}\right)\right)_{\left.\right|_{z=0}}\right)}{q^{n} n !},
$$

where as before, $\mathrm{fp}_{z=0}$ stands for the finite part at $z=0$.

Proof. 1. We write $A(z)=A(z)|D|^{q z}|D|^{-q z}$ and implement a Taylor expansion on the holomorphic family $A(z)|D|^{-q z}$, which has constant order $a$ :

$$
\left.\operatorname{Tr}(A(z))\right|^{\text {mer }}=\sum_{n=0}^{\infty} \frac{z^{n}}{n !} \operatorname{Tr}\left(\left.\left(\left(\partial_{z}^{n}\left(A(z)|D|^{q z}\right)\right)_{\mid z=0}|D|^{-q z}\right)\right|^{\text {mer }}\right) .
$$

By Proposition 2, the higher derivatives $\left(\partial_{z}^{n}\left(A(z)|D|^{q z}\right)\right)_{\left.\right|_{z=0}}$ are operators of order $a$. We have

$$
\begin{aligned}
\left.\operatorname{Res}_{0}^{j+1}(\operatorname{Tr}(A(z)))\right|^{\text {mer }} & =\sum_{n=0}^{\infty} \operatorname{Res}_{0}^{n+j+1} \frac{\left(\operatorname{Tr}\left(\left(\partial_{z}^{n}\left(A(z)|D|^{q z}\right)_{\left.\right|_{z=0}}|D|^{-q z}\right)\right)\right)}{n !} \\
& =\sum_{n=0}^{\infty} \frac{\left.\tau_{n+j}^{|D|}\left(\left(\partial_{z}^{n}\left(A(z)|D|^{q z}\right)\right)_{\left.\right|_{z=0}}\right)\right|^{\text {mer }}}{q^{n+j+1} n !} \\
& =\sum_{n=0}^{k-j} \frac{\tau_{n+j}^{|D|}\left(\left(\partial_{z}^{n}\left(A(z)|D|^{q z}\right)\right)_{\left.\right|_{z=0}}\right)}{q^{n+j+1} n !}
\end{aligned}
$$

since for any abstract pseudodifferential operator $A$, we have $\tau_{k+l}^{|D|}(A)=0 \forall l>0$.

2. The finite part at $z=0$ is obtained from setting $j=-1$ in the previous formula.

Remark 7. These formulae are similar to formulae for the higher residues of traces of holomorphic families of log-polyhomogeneous pseudodifferential operators of logarithmic type $k$, acting on smooth sections of a vector bundle, which were derived in [10]. 


\section{Discrepancies}

As before we assume that $(\mathrm{H}),(\mathrm{Dk})$ and $(\mathrm{T})$ are satisfied.

We want to measure the obstructions preventing the linear forms $\tau_{j}^{|D|}$ from having the expected properties of a trace, which we interpret as discrepancies of the linear forms $\tau_{j}^{|D|}$.

The $|D|$-dependence of the $|D|$-weighted residue trace $\tau_{j}^{|D|}(A)$ of an abstract pseudodifferential operator $A$ is one of these defects. We show how a zero order perturbation $|D| \rightarrow|D|+P$ of the weight $|D|$ by some $P \in \mathcal{E}^{0}(\mathcal{A}, D)$ affects $|D|$-weighted residue traces. We express this variation in terms of expressions of the type $\tau_{j+n}^{|D|}\left(\mathcal{P}_{n}(A,|D|)\right)$, where $\mathcal{P}_{n}(A,|D|)$ are abstract pseudodifferential operators indexed by positive integers $n$.

On the grounds of formulae which hold in the classical setup (see formulae (14) and (15) in [15] with $\phi(x)=x^{-z}$ ), similar to formulae derived in [4] and [6] (see e.g., the proof of Proposition 4.14), for an operator $P \simeq \sum_{j=0}^{\infty} b_{j}|D|^{-j} \in \mathcal{E}^{0}(\mathcal{A}, D)$ and a complex number $z$ we define $(|D|+P)^{-z}$ by

$$
\begin{aligned}
(|D|+P)^{-z}: \simeq|D|^{-z} & \\
& +\sum_{n=1}^{\infty} \sum_{|k|=0}^{\infty}(-1)^{|k|+n} \frac{\Gamma(z+|k|+n)}{\Gamma(z)} \frac{\delta^{k_{1}}(P) \cdots \delta^{k_{n}}(P)|D|^{-z-|k|-n}}{k !\left(k_{1}+1\right)\left(k_{1}+k_{2}+2\right) \cdots\left(k_{1}+\cdots+k_{n}+n\right)},
\end{aligned}
$$

where we have set $k !=k_{1} ! \cdots k_{n}$ ! and $|k|=k_{1}+\cdots+k_{n}$. Note that the real part of the order $-z-|k|-n$ of the operators $\delta^{k_{1}}(P) \cdots \delta^{k_{n}}(P)|D|^{-z-|k|-n}$ decreases with $n$ and $|k|$. Using (2.1), one can write $(|D|+P)^{-z}$ as $\sum_{j=0}^{\infty} \tilde{b}_{j}(z)|D|^{-z-j}$ with $\tilde{b}_{j}(z), j \in \mathbb{Z}_{\geq 0}$ holomorphic in $\mathcal{B}$, so that $D(z):=(|D|+P)^{-z}$ defines a holomorphic family of abstract pseudodifferential operators of order $-z$. It follows from Proposition 3 that for any abstract pseudodifferential operator $A$ acting on $\mathcal{H}^{\infty}$, the map $z \mapsto \operatorname{Tr}\left(A(|D|+P)^{-z}\right)$ is holomorphic on some half-plane and extends to a meromorphic function $\left.\operatorname{Tr}\left(A(|D|+P)^{-z}\right)\right|^{\text {mer }}$ with poles of order $\leq k+1$. Let us set:

$$
\tau_{j}^{|D|+P}(A):=\left.\operatorname{Res}_{0}^{j+1} \operatorname{Tr}\left(A(|D|+P)^{-z}\right)\right|^{\text {mer }} .
$$

Proposition 4. Given $P \simeq \sum_{j=0}^{\infty} b_{j}|D|^{-j}$ in $\mathcal{E}^{0}(\mathcal{A}, D)$, for any non-negative integer $k$ and any $j \in[[-1, k-1]]$

$$
\tau_{j}^{|D|+P}(A)-\tau_{j}^{|D|}(A)=\sum_{n=1}^{k-j} \frac{\tau_{j+n}^{|D|}\left(A\left(\partial_{z}^{n}\left((|D|+P)^{-z}|D|^{z}\right)_{\mid z=0}\right)\right)}{n !}
$$

holds for any operator $A$ in $\mathcal{E}^{a}(\mathcal{A}, D)$ of any complex order a. Moreover,

$$
\tau_{k}(A):=\tau_{k}^{|D|+P}(A)=\tau_{k}^{|D|}(A)
$$

is independent of the perturbation $P$ and when $k=0$ the above formula reads

$$
\tau_{-1}^{|D|+P}(A)-\tau_{-1}^{|D|}(A)=\tau_{0}(A(\log (|D|+P)-\log |D|)) .
$$

Proof. We apply Theorem 1 to the holomorphic family

$$
A(z)=A\left((|D|+P)^{-z}-|D|^{-z}\right)=A\left((|D|+P)^{-z}|D|^{z}-1\right)|D|^{-z},
$$

of order $\alpha(z)=a-z$. 
By Proposition 2, the higher derivatives at zero

$$
\left(\partial_{z}^{n}\left(A(z)|D|^{z}\right)\right)_{\left.\right|_{z=0}}=A \partial_{z}^{n}\left((|D|+P)^{-z}|D|^{z}\right)_{\left.\right|_{z=0}}
$$

have order zero, and by Theorem 1, we have

$$
\begin{aligned}
\tau_{j}^{|D|+P}(A)-\tau_{j}^{|D|}(A) & =\operatorname{Res}_{0}\left(\left.z^{j} \operatorname{Tr}\left(A\left((|D|+P)^{-z}-|D|^{-z}\right)\right)\right|^{\text {mer }}\right) \\
& =\operatorname{Res}_{0}^{j+1}\left(\left.\operatorname{Tr}\left(A\left((|D|+P)^{-z}-|D|^{-z}\right)\right)\right|^{\text {mer }}\right) \\
& =\sum_{n=0}^{k-j} \frac{\tau_{j+n}^{|D|}\left(A\left(\left.\partial_{z}^{n}\left((|D|+P)^{-z}|D|^{z}-1\right)\right|_{z=0}\right)\right)}{n !} \\
& =\sum_{n=1}^{k-j} \frac{\tau_{j+n}^{|D|}\left(A\left(\left.\partial_{z}^{n}(|D|+P)^{-z}|D|^{z}\right|_{\left.\right|_{z=0}}\right)\right)}{n !} \quad \text { if } \quad j<k,
\end{aligned}
$$

since the $n=0$ term vanishes. If $j=k$, we have $\tau_{j}^{|D|+P}(A)-\tau_{j}^{|D|}(A)=0$, which shows the invariance of $\tau_{k}^{|D|}$ under a perturbation $P$. By Proposition 1, the operator $A(\log (|D|+P)-\log |D|)$ lies in $\mathcal{E}^{a}(\mathcal{A}, D)$ and when $k=0$, the above computation yields

$$
\tau_{-1}^{|D|+P}(A)-\tau_{-1}^{|D|}(A)=\tau_{0}(A(\log |D|-\log (|D|+P))) .
$$

Remark 8. When $k=0$, the linear form $\tau_{0}$ generalises Wodzicki's noncommutative residue $[22,23]$ on classical pseudodifferential operators acting on smooth sections of a vector bundle $E$ over a closed manifold $M$ (see [8] for a review).

The linear form $\tau_{j}^{|D|}$ does not vanish on commutators as could be expected of a trace, leading to another discrepancy. The following result yields back Proposition II.1 in [4].

Proposition 5. Given two abstract pseudodifferential operators $A, B$ we have:

$$
\tau_{j}^{D}([A, B])=\sum_{n=1}^{k-j}(-1)^{n+1} \frac{\tau_{j+n}^{|D|}\left(A L^{n}(B)\right)}{n !}, \quad \forall j \in[[-1, k-1]],
$$

where we have set $L(A):=[\log |D|, A]$.

Moreover $\tau_{k}([A, B])=0$ and when $k=0$ the above formula reads:

$$
\tau_{-1}^{D}([A, B])=\tau_{0}(A([\log |D|, B])) .
$$

Proof. Using the cyclic property of the usual trace Tr, by analytic continuation we have:

$$
\begin{aligned}
\left.\operatorname{Tr}\left(B A|D|^{-z}\right)\right|^{\text {mer }} & =\left.\operatorname{Tr}\left(\left(|D|^{-z / 2} B\right)\left(A|D|^{-z / 2}\right)\right)\right|^{\text {mer }} \\
& =\left.\operatorname{Tr}\left(\left(A|D|^{-z / 2}\right)\left(|D|^{-z / 2} B\right)\right)\right|^{\text {mer }}=\left.\operatorname{Tr}\left(A|D|^{-z} B\right)\right|^{\text {mer }} .
\end{aligned}
$$

Hence,

$$
\left.\operatorname{Tr}\left(A B|D|^{-z}-B A|D|^{-z}\right)\right|^{\text {mer }}=\left.\operatorname{Tr}\left(A B|D|^{-z}-A|D|^{-z} B\right)\right|^{\text {mer }}=\left.\operatorname{Tr}\left(A\left[B,|D|^{-z}\right]\right)\right|^{\text {mer }} .
$$

We can apply Theorem 1 to the holomorphic family

$$
A(z)=A\left[B,|D|^{-z}\right] .
$$

Indeed,

$$
A(z)|D|^{z}=A B-A|D|^{-z} B|D|^{z}=A(B-\sigma(z)(B)),
$$


where we have set $\sigma(z)(A)=|D|^{-z} A|D|^{z}$. Since

$$
|D|^{-z} A \simeq A|D|^{-z}+\sum_{k=1}^{\infty} c_{-z, k} \delta^{k}(A)|D|^{-z-k},
$$

it follows that

$$
\sigma(z)(A) \simeq A+\sum_{k=1}^{\infty} c_{-z, k} \delta^{k}(A)|D|^{-k},
$$

where as before $c_{\alpha, k}=\frac{\alpha(\alpha-1) \cdots(\alpha-k+1)}{k !}$ for any positive integer $k$. By Proposition 2, the higher derivatives at zero $\partial_{z}^{n}\left(A(z)|D|^{z}\right)_{\mid z=0}$ have order $a+b$, where $a$ is the order of $A$ and $b$ that of $B$.

Since $\partial_{z}^{n} \sigma(z)(A)_{\left.\right|_{z=0}}=(-1)^{n} L(A)$, Theorem 1 yields

$$
\begin{aligned}
\tau_{j}^{|D|}([A, B]) & =\sum_{n=0}^{k-j} \frac{\tau_{j+n}^{|D|}\left(\partial_{z}^{n}\left(A(z)|D|^{z}\right)\right)_{\left.\right|_{z=0}}}{n !}=-\sum_{n=0}^{k-j} \frac{\tau_{j+n}^{|D|}\left(A\left(\partial_{z}^{n} \sigma(z)(B)\right)\right)_{\mid z=0}}{n !} \\
& =\sum_{n=0}^{k-j}(-1)^{n+1} \frac{\tau_{j+n}^{|D|}\left(A L^{n}(B)\right)}{n !}=\sum_{n=1}^{k-j}(-1)^{n+1} \frac{\tau_{j+n}^{|D|}\left(A L^{n}(B)\right)}{n !} \quad \text { if } \quad j<k
\end{aligned}
$$

since the $n=0$ term vanishes.

This computation also shows that $\tau_{k}([A, B])=\tau_{k}^{|D|}([A, B])=0$. When $k=0$ it shows that:

$$
\tau_{-1}^{|D|}([A, B])=\tau_{0}(A([\log |D|, B])) .
$$

These discrepancy formulae are similar to the anomaly formulae for weighted traces of classical pseudodifferential operators, see [11, 3, 16] and [14] for an extension to log-polyhomogeneous operators.

\section{$7 \quad$ An analog of Kontsevich and Vishik's canonical trace}

In [9] Kontsevich and Vishik popularised what is known as the canonical trace on non-integer order classical pseudodifferential operators acting on smooth sections of a vector bunlde $E$ over a closed manifold $M$. This linear form which vanishes on commutators of non-integer order classical pseudodifferential operators, is the unique (up to a multiplicative constant) linear extension of the ordinary trace to the set of non-integer order operators with that property. The non integrality assumption on the order can actually be weakened to the order not lying in the set $[-n,+\infty[\cap \mathbb{Z}$ which corresponds to $-\mathrm{Sd}$, with $\mathrm{Sd}$ the dimension spectrum of the spectral triple $\left(C^{\infty}(M), L^{2}(M, E), D\right)$.

For a spectral triple which fulfills assumptions $(\mathrm{H}),(\mathrm{Dk})$ and $(\mathrm{T})$, we replace this assumption on the order with the requirement that the order lies outside the set $-\mathrm{Sd}$.

Lemma 2. For an abstract pseudodifferential operator $A$ whose order a lies outside the set -Sd and for any holomorphic family $A(z)$ such that $A(0)=A$, the map $\left.z \mapsto \operatorname{Tr}(A(z))\right|^{\text {mer }}$ is holomorphic at $z=0$.

In particular, the map $\left.z \mapsto \operatorname{Tr}\left(A|D|^{-z}\right)\right|^{\text {mer }}$ is holomorphic at zero so that

$$
\tau_{j}^{|D|}(A)=0 \quad \forall j \in \mathbb{Z}_{+} \quad \text { and } \quad \tau_{-1}^{|D|}(P)=\lim _{z \rightarrow 0}\left(\left.\operatorname{Tr}\left(A|D|^{-z}\right)\right|^{\text {mer }}\right) .
$$


Proof. In view of the asymptotic expansion $A(z) \simeq \sum_{j=0}^{\infty} b_{j}(z)|D|^{\alpha(z)-j}$, where $\alpha(z)$ is the order of the holomorphic family $A(z)$, it suffices to show the result for each holomorphic family $A_{j}(z)=b_{j}(z)|D|^{\alpha(z)-j}$, with $j$ varying in $\mathbb{Z}_{\geq 0}$. Such a family has order $\alpha_{j}(z)=\alpha(z)-j$ so that the poles of the meromorphic map $\left.z \mapsto \operatorname{Tr}\left(A_{j}(z)\right)\right|^{\text {mer }}$ lie in $\alpha^{-1}\left(-\operatorname{Sd}+\mathbb{Z}_{\geq 0}\right) \subset \alpha^{-1}(-\operatorname{Sd})$, where we have used assumption (T) on the spectrum. Since the order of $A$ does not lie in -Sd, 0 does not lie in this set of poles, which leads to the two identities in the statement.

Proposition 6. For an abstract pseudodifferential operator A whose order a does not lie in-Sd,

$$
\tau_{-1}(A):=\tau_{-1}^{|D|}(A)
$$

is insensitive to lower order perturbations $|D| \rightarrow|D|+P$ of the weight $|D|$, with $P \simeq \sum_{j=0}^{\infty} b_{j}|D|^{-j}$ in $\mathcal{E}^{0}(\mathcal{A}, D)$.

Proof. By (6.2) and for $j$ in $[[-1, k-1]]$

$$
\begin{aligned}
\tau_{-1}^{|D|+P}(A)-\tau_{-1}^{|D|}(A) & =\sum_{n=1}^{k+1} \frac{\tau_{-1+n}^{|D|}\left(A\left(\partial_{z}^{n}\left((|D|+P)^{-z}|D|^{z}\right)_{z=0}\right)\right)}{n !} \\
& =\sum_{m=0}^{k} \frac{\tau_{m}^{|D|}\left(A\left(\partial_{z}^{m+1}\left((|D|+P)^{-z}|D|^{z}\right)_{\left.\right|_{z=0}}\right)\right)}{(m+1) !} .
\end{aligned}
$$

By Proposition 2, the operators $A\left(\left.\partial_{z}^{m+1}\left((|D|+P)^{-z}|D|^{z}\right)\right|_{z=0}\right), m \in \mathbb{Z}_{\geq 0}$, all have orders $a \notin$ -Sd, as a consequence of which all the terms $\tau_{m}^{|D|}\left(A\left(\partial_{z}^{m+1}\left((|D|+P)^{-z}|D|^{z}\right)_{\left.\right|_{z=0}}\right)\right)$ in the sum vanish. Thus,

$$
\tau_{-1}^{|D|+P}(A)=\tau_{-1}^{|D|}(A) .
$$

The linear form $\tau_{-1}$ provides a substitute on abstract pseudodifferential operators with order outside the discrete set $-\mathrm{Sd}$, for the canonical trace TR defined on classical pseudodifferential operators, whose order lies outside the set $[-n,+\infty[\cap \mathbb{Z}$.

Proposition 7. The linear ${ }^{8}$ map $\tau_{-1}$ vanishes on commutators, whose order does not lie in-Sd:

$$
(\operatorname{ord}(A)+\operatorname{ord}(B) \notin-\mathrm{Sd}) \Longrightarrow \tau_{-1}([A, B])=0 .
$$

Proof. By (6.3) we have

$$
\tau_{-1}([A, B])=\sum_{n>0}(-1)^{n+1} \frac{\tau_{n-1}^{|D|}\left(A L^{n}(B)\right)}{n !}=\sum_{m \geq 0}(-1)^{m} \frac{\tau_{m}^{|D|}\left(A L^{m+1}(B)\right)}{(m+1) !} .
$$

The orders of the operators $A L^{m+1}(B)$, which lie in $\operatorname{ord}(A)+\operatorname{ord}(B)-\mathbb{Z}_{\geq 0}$ do not lie in - Sd since $\operatorname{ord}(A)+\operatorname{ord}(B)$ does not and since by assumption $(\mathrm{T})$ we have the inclusion: $-\mathrm{Sd} \subset-\mathrm{Sd}-\mathbb{Z}_{>0}$. Hence, the terms $\tau_{m}^{|D|}\left(A L^{m+1}(B)\right), m \in \mathbb{Z}_{\geq 0}$ in the sum vanish and $\tau_{-1}([A, B])=0$.

Thus, the linear map $\tau_{-1}$ defines a "trace" on the set of operators, whose order lies outside the set -Sd in the same way that Kontsevich and Vishik's canonical trace TR defines a "trace" on non-integer order classical pseudodifferential operators.

\footnotetext{
${ }^{8}$ Meaning by this that it acts on linear combinations of operators whose order does not lie in $-\mathrm{Sd}$.
} 


\section{Acknowledgements}

I thank the Australian National University in Canberra for its hospitality during a ten days stay, when this work was initiated and Bai Ling Wang for inviting me there, as well as for stimulating workshops he organised and the lively discussions that followed. I am grateful to Alan Carey for helping organise my stay and I thank him and Adam Rennie most warmly for various informal discussions we had during my stay, which triggered this work. I would also like to acknowledge the referees' substantial help in improving of this paper.

\section{References}

[1] Berline N., Getzler E., Vergne M., Heat kernels and Dirac operators, Springer-Verlag, Berlin, 2004.

[2] Connes A., Noncommutative geometry, Academic Press, San Diego, CA, 1994.

[3] Cardona A., Ducourtioux C., Magnot J.P., Paycha S., Weighted traces on algebras of pseudo-differential operators and geometry on loop groups, Infin. Dimens. Anal. Quantum Probab. Relat. Top. 5 (2002), 503540, math.OA/0001117.

[4] Connes A., Moscovici H., The local index formula in noncommutative geometry, Geom. Funct. Anal. 5 (1995), 174-243.

[5] Dunford N., Schwartz J.T., Linear operators. Part III. Spectral operators, John Wiley \& Sons, Inc., New York, 1988.

[6] Higson N., The residue index theorem of Connes and Moscovici, in Surveys in Noncommutative Geometry, Clay Math. Proc., Vol. 6, Amer. Math. Soc., Providence, RI, 2006, 71-126.

[7] Hörmander L., The analysis of linear partial differential operators. I. Distribution theory and Fourier analysis, Grundlehren der Mathematischen Wissenschaften, Vol. 256, Springer-Verlag, Berlin, 1983.

[8] Kassel Ch., Le résidu non commutatif (d'après M. Wodzicki), Astérisque no. 177-178 (1989), exp. no. 708, 199-229.

[9] Kontsevich M., Vishik S., Geometry of determinants of elliptic operators, in Functional Analysis on the Eve of the 21st Century, Vol. 1 (New Brunswick, NJ, 1993), Progr. Math., Vol. 131, Birkhäuser Boston, Boston, MA, 1995, 173-197, hep-th/9406140.

Kontsevich M., Vishik S., Determinants of elliptic pseudo-differential operators, hep-th/9404046.

[10] Lesch M., On the noncommutative residue for pseudodifferential operators with log-polyhomogeneous symbols, Ann. Global Anal. Geom. 17 (1998), 151-187, dg-ga/9708010.

[11] Melrose R., Nistor V., Homology of pseudo-differential operators. I. Manifolds with boundary, funct-an/9606005.

[12] Maniccia L., Schrohe E., Seiler J., Uniqueness of the Kontsevich-Vishik trace, Proc. Amer. Math. Soc. 136 (2008), 747-752, math.FA/0702250.

[13] Okikiolu K., The Campbell-Hausdorff theorem for elliptic operators and a related trace formula, Duke Math. J. 79 (1995), 687-722.

[14] Paycha S., Regularised integrals, sums and traces; an analytic point of view, Monograph in preparation.

[15] Paycha S., Noncommutative Taylor expansions and second quantised regularised traces, in Combinatorics and Physics, Clay Math. Proc., to appear.

[16] Paycha S., Scott S., A Laurent expansion for regularised integrals of holomorphic symbols, Geom. Funct. Anal. 17 (2007), 491-536, math.AP/0506211.

[17] Seeley R.T., Complex powers of an elliptic operator, in Singular Integrals (Proc. Sympos. Pure Math., Chicago, Ill., 1966), Amer. Math. Soc., Providence, R.I., 1967, 288-307.

[18] Shubin M.A., Pseudodifferential operators and spectral theory, 2nd ed., Springer-Verlag, Berlin, 2001.

[19] Taylor M.E., Pseudodifferential operators, Princeton Mathematical Series, Vol. 34, Princeton University Press, Princeton, N.J., 1981.

[20] Trèves F., Introduction to pseudodifferential and Fourier integral operators, Vol. 1. Pseudodifferential operators, The University Series in Mathematics, Plenum Press, New York - London, 1980. 
[21] Varilly J.C., An introduction to noncommutative geometry, EMS Series of Lectures in Mathematics, European Mathematical Society (EMS), Zürich, 2006.

[22] Wodzicki M., Spectral asymmetry and noncommutative residue, PhD Thesis, Steklov Mathematics Institute, Moscow, 1984 (in Russian).

[23] Wodzicki M., Noncommutative residue. I. Fundamentals, in K-Theory, Arithmetic and Geometry (Moscow, 1984-1986), Lecture Notes in Math., Vol. 1289, Springer, Berlin, 1987, 320-399. 